\title{
The Local Time of the Fractional Ornstein-Uhlenbeck Process
}

\author{
Guangjun Shen, Dongjin Zhu, Yong Ren, and Xueping Ding \\ Department of Mathematics, Anhui Normal University, 1 East Beijing Road, Wuhu 241000, China \\ Correspondence should be addressed to Guangjun Shen; gjshen@163.com
}

Received 11 June 2013; Revised 11 August 2013; Accepted 11 August 2013

Academic Editor: Mark McKibben

Copyright (C) 2013 Guangjun Shen et al. This is an open access article distributed under the Creative Commons Attribution License, which permits unrestricted use, distribution, and reproduction in any medium, provided the original work is properly cited.

We investigate the Hölder regularity of the local time of the fractional Ornstein-Uhlenbeck process $X^{H}=\left\{X_{t}^{H}, t \geq 0\right\}$. As a related problem, we study the collision local time of two independent fractional Ornstein-Uhlenbeck $X^{H_{i}}=\left\{X_{t}^{H_{i}}, t \geq 0\right\}, i=1,2$ with respective indices $H_{1}, H_{2} \in(0,1)$.

\section{Introduction}

The Brownian motion and the Ornstein-Uhlenbeck process are the two most well-studied and widely applied stochastic processes. The Einstein-Smoluchowski theory may be seen as an idealized Ornstein-Uhlenbeck theory, and predictions of either cannot be distinguished by the experiment. However, if the Brownian particle is under the influence of an external force, the Einstein-Smoluchowski theory breaks down, while the Ornstein-Uhlenbeck theory remains successful. It is well known that a diffusion process $X=\left(X_{t}\right)_{t>0}$ starting from $x \in$ $\mathbb{R}$ is called Ornstein-Uhlenbeck process with coefficients $v>$ 0 if its infinitesimal generator is

$$
L=\frac{1}{2} v^{2} \frac{d^{2}}{d x^{2}}-x \frac{d}{d x} .
$$

The Ornstein-Uhlenbeck process (see, e.g., Revuz and Yor [1]) has a remarkable history in physics. It is introduced to model the velocity of the particle diffusion process, and later it has been heavily used in finance, and thus in econophysics. It can be constructed as the unique strong solution of Itô stochastic differential equation

$$
d X_{t}=-X_{t} d t+v d B_{t}, \quad X_{0}=x,
$$

where $B$ is a standard Brownian motion starting at 0 .

Recently, as an extension of Brownian motion, fractional Brownian motion has become an object of intense study, due to its interesting properties and its applications in various scientific areas including condensed matter physics, biological physics, telecommunications, turbulence, image processing, finance, and econophysics (see, e.g., Gouyet [2], Nualart [3],
Biagini et al. [4], Mishura [5], Willinger et al. [6], and references therein). Recall that fractional Brownian motion $B^{H}$ with Hurst index $H \in(0,1)$ is a central Gaussian process with $B_{0}^{H}=0$ and the covariance function

$$
E\left[B_{t}^{H} B_{s}^{H}\right]=\frac{1}{2}\left[t^{2 H}+s^{2 H}-|t-s|^{2 H}\right]
$$

for all $t, s \geqslant 0$. This process was first introduced by Kolmogorov and studied by Mandelbrot and van Ness [7], where a stochastic integral representation in terms of a standard Brownian motion was established. For $H=1 / 2, B^{H}$ coincides with the standard Brownian motion $B . B^{H}$ is neither a semimartingale nor a Markov process unless $H=1 / 2$, and so many of the powerful techniques from stochastic analysis are not available when dealing with $B^{H}$. It has self-similar, longrange dependence, Hölder paths, and it has stationary increments. These properties make $B^{H}$ an interesting tool for many applications.

On the other hand, extensions of the classical OrnsteinUhlenbeck process have been suggested mainly on demand of applications. The fractional Ornstein-Uhlenbeck process is an extension of the Ornstein-Uhlenbeck process, where fractional Brownian motion is used as integrator

$$
d X_{t}=-X_{t} d t+v d B_{t}^{H}, \quad X_{0}=x .
$$

Then (4) has a unique solution $X_{t}^{H}=\left\{X_{t}^{H}, 0 \leq t \leq T\right\}$, which can be expressed as

$$
X_{t}^{H}=e^{-t}\left(x+v \int_{0}^{t} e^{s} d B_{s}^{H}\right)
$$


and the solution is called the fractional Ornstein-Uhlenbeck process. More work for the process can be found in Cheridito et al. [8], Lim and Muniandy [9], Metzler and Klafter [10], and Yan et al. $[11,12]$. Clearly, when $H=1 / 2$, the fractional Ornstein-Uhlenbeck process is the classical OrnsteinUhlenbeck process $X$ with parameter $v$ starting at $x \in \mathbb{R}$. An advantage of using fractional Ornstein-Uhlenbeck process is to realize stationary long range dependent processes.

The intuitive idea of local time $L(t, x)$ for a stochastic process $X$ is that $L(t, x)$ measures the amount of time $X$ spends at the level $x$ during the interval $[0, t]$. Moreover, since the work of Varadhan [13], the local time of stochastic processes has become an important subject. Therefore, it seems interesting to study the local time of fractional Ornstein-Uhlenbeck process, a rather special class of Gaussian processes.

In this paper, we focus our attention on the Hölder regularity of the local time of fractional Ornstein-Uhlenbeck process.

The rest of this paper is organized as follows. Section 2 contains a brief review on the local times of Gaussian processes and the approach of chaos expansion of the Gaussian process. In Section 3, we give Hölder regularity of the local time. In Section 4, as a related problem, we study the so-called collision local time of two independent fractional OrnsteinUhlenbeck $X^{H_{i}}=\left\{X_{t}^{H_{i}}, t \geq 0\right\}, i=1,2$ with respective indices $H_{1}, H_{2} \in(0,1)$.

\section{Preliminaries}

2.1. Local Times and Local Nondeterminism. We recall briefly the definition of local time. For a comprehensive survey on local times of both random and nonrandom vector fields, we refer to Alder [14], Geman and Horowitz [15], and Xiao [1618 ]. Let $X(t)$ be any Borel function on $\mathbb{R}$ with values in $\mathbb{R}$. For any Borel set $B \subset \mathbb{R}$, the occupation measure of $X$ is defined by

$$
\mu_{B}(A)=\lambda_{1}\{t \in B, X(t) \in A\},
$$

for all Borel set $A \subseteq \mathbb{R}$, where $\lambda_{1}$ is the one-dimensional Lebesgue measure. If $\mu_{B}$ is absolutely continuous with respect to the Lebesgue measure $\lambda_{1}$ on $\mathbb{R}$, we say that $X(t)$ has a local time on $B$ and define its local time $L(B, x)$ to be the RadonNikodym derivative of $\mu_{B}$. If $B=[0, t]$, we simply write $L(B, x)$ as $L(t, x)$. If $I=[0, T]$ and $L(t, x)$ is continuous as a function of $(t, x) \in I \times \mathbb{R}$, then we say that $X$ has a jointly continuous local time on $I$. In this latter case, the set function $L(\cdot, x)$ can be extended to be a finite Borel measure on the level set (see Adler [14, Theorem 8.6.1])

$$
X_{I}^{-1}(x)=\{t \in I: X(t)=x\}
$$

This fact has been used by many authors to study fractal properties of level sets, inverse image, and multiple times of stochastic processes. For example, Xiao [16] and $\mathrm{Hu}$ [19] have studied the Hausdorff dimension, and exact Hausdorff and packing measure of the level sets of iterated Brownian motion, respectively.
For a fixed sample function at fixed $t$, the Fourier transform on $x$ of $L(t, x)$ is the function

$$
f(t, u)=\int_{\mathbb{R}} e^{i u x} L(t, x) d x .
$$

Using the density of occupation formula we have

$$
f(t, u)=\int_{0}^{t} e^{i u X(s)} d s
$$

We can express the local times $L(t, x)$ as the inverse Fourier transform of $f(t, u)$, namely,

$$
L(t, x)=\frac{1}{2 \pi} \int_{-\infty}^{+\infty}\left(\int_{0}^{t} e^{i u(X(s)-x)} d s\right) d u .
$$

It follows from (10) that for any $x, y \in \mathbb{R}, t, t+\omega \in[0, T]$ and any integer $n \geq 2$, we have (see, e.g., Boufoussi et al. [20, 21])

$$
\begin{gathered}
E(L(t+h, x)-L(t, x))^{n} \\
=\frac{1}{(2 \pi)^{n}} \int_{[t, t+\omega]^{n}} \int_{\mathbb{R}^{n}} e^{-i x \sum_{j=1}^{n} u_{j}} E\left(e^{i \sum_{j=1}^{n} u_{j} X\left(s_{j}\right)}\right) \\
\times \prod_{j=1}^{n} d u_{j} \prod_{j=1}^{n} d s_{j},
\end{gathered}
$$

and for every even integer $n \geq 2$,

$$
\begin{aligned}
E(L(t+h, x)-L(t, x) & -L(t+h, y)+L(t, y))^{n} \\
=\frac{1}{(2 \pi)^{n}} \int_{[t, t+\omega]^{n}} \int_{\mathbb{R}^{n}} & \prod_{j=1}^{n}\left[e^{-i x u_{j}}-e^{-i y u_{j}}\right] \\
& \times E\left(e^{i \sum_{j=1}^{n} u_{j} X\left(s_{j}\right)}\right) \prod_{j=1}^{n} d u_{j} \prod_{j=1}^{n} d s_{j} .
\end{aligned}
$$

The concept of local nondeterminism was first introduced by Berman [22] to unify and extend his methods for studying local times of real-valued Gaussian processes. Let $X=\left\{X(t), t \in \mathbb{R}_{+}\right\}$be a real-valued, separable Gaussian process with mean 0 and let $T \subset \mathbb{R}_{+}$be an open interval. Assume that $E\left[X(t)^{2}\right]>0$ for all $t \in T$ and there exists $\delta>0$ such that

$$
E\left[(X(s)-X(t))^{2}\right]>0,
$$

for $s, t \in T$ with $0<|s-t|<\delta$.

Recall from Berman [22] that $X$ is called locally nondeterministic on $T$ if for every integer $n \geq 2$,

$$
\lim _{\epsilon \rightarrow 0} \inf _{t_{n}-t_{1} \leq \epsilon} V_{n}>0,
$$

where $V_{n}$ is the relative prediction error as follows:

$$
V_{n}=\frac{\operatorname{Var}\left(X\left(t_{n}\right)-X\left(t_{n-1}\right) \mid X\left(t_{1}\right), \ldots, X\left(t_{n-1}\right)\right)}{\operatorname{Var}\left(X\left(t_{n}\right)-X\left(t_{n-1}\right)\right)},
$$

and the infimum in (14) is taken over all ordered points $t_{1}<$ $t_{2}<\cdots<t_{n}$ in $T$ with $t_{n}-t_{1} \leq \epsilon$. Roughly speaking, (14) 
means that a small increment of the process $X$ is not almost relatively predictable based on a finite number of observations from the immediate past.

It follows from Berman [22, Lemma 2.3] that (14) is equivalent to the following property which says that $X$ has locally approximately independent increments: for any positive integer $n \geq 2$, there exist positive constants $C_{n}$ and $\delta$ (both may depend on $n$ ) such that

$$
\begin{aligned}
& \operatorname{Var}\left(\sum_{j=1}^{n} u_{j}\left[X\left(t_{j}\right)-X\left(t_{j-1}\right)\right]\right) \\
& \quad \geq C_{n} \sum_{j=1}^{n} u_{j}^{2} \operatorname{Var}\left[X\left(t_{j}\right)-X\left(t_{j-1}\right)\right],
\end{aligned}
$$

for all ordered points $0=t_{0}<t_{1}<t_{2}<\cdots<t_{n}$ in $T$ with $t_{n}-t_{1}<\delta$ and all $u_{j} \in \mathbb{R}(1 \leq j \leq n)$. We refer to Nolan [23, Theorem 2.6] for a proof of the above equivalence in a much more general setting.

For simplicity throughout this paper we let $C_{n}$ stand for a positive constant depending only on the subscripts and its value may be different in different appearances, and this assumption is also adaptable to $C, C_{H}$.

2.2. Chaos Expansion. Let $\Omega$ be the space of continuous $\mathbb{R}^{1}$ valued functions $\omega$ on $[0, T]$. Then $\Omega$ is a Banach space with respect to the supreme norm. Let $\mathscr{F}$ be the $\sigma$-algebra on $\Omega$. Let $P$ be the probability measure on the measurable space $(\Omega, \mathscr{F})$. Let $\mathbb{E}$ denote the expectation on this probability space. The set of all square integrable functionals is denoted by $L^{2}(\Omega, P)$, that is,

$$
E\left(F^{2}\right)=\int_{\Omega}|F(\omega)|^{2} P(d \omega)<\infty .
$$

We can introduce the chaos expansion, which is an orthogonal decomposition of $L^{2}(\Omega, P)$. We refer to $\mathrm{Hu}$ [24], Nualart [3], and the references therein for more details. Let $X:=\left\{X_{t}, t \in[0, T]\right\}$ be a Gaussian process defined on the probality space $(\Omega, \mathscr{F}, P)$. If $p_{n}(x)$ is a polynomial of degree $n$ in $x$, then we call $p_{n}\left(X_{t}\right)$ a polynomial function of $X$ with $t \epsilon$ $[0, T]$. Let $\mathscr{P}_{n}$ be the completion with respect to the $L^{2}(\Omega, P)$ norm of the set $\left\{p_{m}\left(X_{t}\right): 0 \leq m \leq n, t \in[0, T]\right\}$. Clearly, $\mathscr{P}_{n}$ is a subspace of $L^{2}(\Omega, P)$. If $\mathscr{C}_{n}$ denotes the orthogonal complement of $\mathscr{P}_{n-1}$ in $\mathscr{P}_{n}$, then $L^{2}(\Omega, P)$ is actually the direct sum of $\mathscr{C}_{n}$, that is,

$$
L^{2}(\Omega, P)=\bigoplus_{n=0}^{\infty} \mathscr{C}_{n}
$$

Namely, for any functional $F \in L^{2}(\Omega, P)$, there are $F_{n}$ in $\mathscr{C}_{n}$, $n=0,1,2, \ldots$, such that

$$
F=\sum_{n=0}^{\infty} F_{n}
$$

The decomposition equation (19) is called the chaos expansion of $F$, and $F_{n}$ is called the $n$th chaos of $F$. Clearly, we have

$$
E\left(|F|^{2}\right)=\sum_{n=0}^{\infty} E\left(\left|F_{n}\right|^{2}\right) \text {. }
$$

Recall that Meyer-Watanabe test function space $\mathcal{U}$ (see Watanabe [25]) is defined as

$$
\mathcal{U}:=\left\{F \in L^{2}(\Omega, P): F=\sum_{n=0}^{\infty} F_{n}, \sum_{n=0}^{\infty} n E\left(\left|F_{n}\right|^{2}\right)<\infty\right\},
$$

and $F \in L^{2}(\Omega, P)$ is said to be smooth if $F \in \mathcal{U}$.

Now, for $F \in L^{2}(\Omega, P)$, we define an operator $\Gamma_{u}$ with $u \in$ $[0,1]$ by

$$
\Gamma_{u} F:=\sum_{n=0}^{\infty} u^{n} F_{n}
$$

Set $\Theta(u):=\Gamma_{\sqrt{u}} F$. Then $\Theta(1)=F$. Define $\Phi_{\Theta}(u):=(d / d u)$ $\left(\|\Theta(u)\|^{2}\right)$, where $\|F\|^{2}:=E\left(|F|^{2}\right)$ for $F \in L^{2}(\Omega, P)$. We have

$$
\Phi_{\Theta}(u)=\sum_{n=1}^{\infty} n u^{n-1} E\left(\left|F_{n}\right|^{2}\right)
$$

Note that $\|\Theta(u)\|^{2}=E\left(|\Theta(u)|^{2}\right)=\sum_{n=0}^{\infty} E\left(u^{n}\left|F_{n}\right|^{2}\right)$.

Proposition 1. Let $F \in L^{2}(\Omega, P)$. Then $F \in \mathcal{U}$ if and only if $\Phi_{\Theta}(1)<\infty$.

Consider two independent fractional Ornstein-Uhlenbeck $X^{H_{i}}=\left\{X_{t}^{H_{i}}, t \geq 0\right\}, i=1,2$, with respective indices $H_{i} \in(0,1)$. Let $H_{n}(x)$ and $x \in \mathbb{R}$ be the Hermite polynomials of degree $n$. That is,

$$
H_{n}(x)=(-1)^{n} \frac{1}{n !} e^{x^{2} / 2} \frac{\partial^{n}}{\partial x^{n}} e^{-x^{2} / 2}
$$

Then,

$$
e^{t x-t^{2} / 2}=\sum_{n=0}^{\infty} t^{n} H_{n}(x)
$$

for all $t \in \mathscr{C}$ and $x \in \mathbb{R}$, this implies that

$$
\begin{aligned}
& \exp \left(i u \xi\left(X_{t}^{H_{1}}-X_{t}^{H_{2}}\right)+\frac{1}{2} u^{2} \xi^{2} \operatorname{Var}\left(X_{t}^{H_{1}}-X_{t}^{H_{2}}\right)\right) \\
& =\sum_{n=0}^{\infty}(i u)^{n} \sigma^{n}(t, \xi) H_{n}\left(\frac{\xi\left(X_{t}^{H_{1}}-X_{t}^{H_{2}}\right)}{\sigma(t, \xi)}\right)
\end{aligned}
$$

where $i=\sqrt{-1}$ and $\sigma(t, \xi)=\sqrt{\operatorname{Var}\left(X_{t}^{H_{1}}-X_{t}^{H_{2}}\right) \xi^{2}}$ for $\xi \in \mathbb{R}$. Because of the orthogonality of $\left\{H_{n}(x), x \in \mathbb{R}\right\}_{n \in \mathbb{Z}_{+}}$, we will get from (19) that

$$
(i u)^{n} \sigma^{n}(t, \xi) H_{n}\left(\frac{\xi\left(X_{t}^{H_{1}}-X_{t}^{H_{2}}\right)}{\sigma(t, \xi)}\right)
$$

is the $n$th chaos of $\exp \left(i u \xi\left(X_{t}^{H_{1}}-X_{t}^{H_{2}}\right)+(1 / 2) u^{2} \xi^{2} \operatorname{Var}\left(X_{t}^{H_{1}}-\right.\right.$ $\left.B_{t}^{H_{2}}\right)$ ) for all $t \geq 0$. 


\section{Local Time of Fractional Ornstein-Uhlenbeck Process}

In this section, we offer the Hölder regularity of the local time of fractional Ornstein-Uhlenbeck process.

Theorem 2. Let $\left\{X_{t}^{H}, t \geq 0\right\}$ be the fractional Ornstein-Uhlenbeck process. Then, for every $t \in \mathbb{R}^{+}$and any $x \in \mathbb{R}$, there exist positive and finite constants $C_{1}$ and $C_{2}$ such that

$$
\begin{aligned}
& \limsup _{h \rightarrow 0} \sup _{x} \frac{L(t+h, x)-L(t, x)}{h^{1-H}\left(\log \log \left(h^{-1}\right)\right)^{H}} \leq C_{1} \quad \text { a.s. } \\
& \limsup _{h \rightarrow 0} \sup _{x, t} \frac{L(t+h, x)-L(t, x)}{h^{1-H}\left(\log \left(h^{-1}\right)\right)^{H}} \leq C_{2} \quad \text { a.s. }
\end{aligned}
$$

Proof. Let $t \geq 0$ be a fixed point. Following the Fourier analytic approach of Berman [26], we have

$$
\begin{aligned}
& E[L(t+h, x)-L(t, x)]^{n} \\
& =\frac{1}{(2 \pi)^{n}} \\
& \quad \times \int_{[t, t+h]^{n}} \int_{\mathbb{R}^{n}} E\left(\exp \left(i \sum_{j=1}^{n} u_{j}\left(X_{s_{j}}^{H}-X_{t}^{H}\right)\right)\right) \\
& \quad \times \prod_{j=1}^{n} d u_{j} \prod_{j=1}^{n} d s_{j} .
\end{aligned}
$$

Let $\Delta X_{s}^{H}=X_{s}^{H}-X_{t}^{H}, s \geq 0$, and denote by $R\left(s_{1}, s_{2}, \ldots, s_{n}\right)$ the covariance matrix of $\left(\Delta X_{s_{1}}^{H}, \ldots, \Delta X_{s_{n}}^{H}\right)$ for different $s_{1}, \ldots, s_{n}$, then we have

$$
\begin{aligned}
& \operatorname{det} R\left(s_{1}, s_{2}, \ldots, s_{n}\right) \\
& =\operatorname{Var}\left(\Delta X_{s_{1}}^{H}\right) \operatorname{Var}\left(\Delta X_{s_{2}}^{H} \mid \Delta X_{s_{1}}^{H}\right) \ldots \\
& \quad \operatorname{Var}\left(\Delta X_{s_{n}}^{H} \mid \Delta X_{s_{1}}^{H}, \ldots, \Delta X_{s_{n-1}}^{H}\right) .
\end{aligned}
$$

By Yan et al. [11], one can write the fractional Ornstein-Uhlenbeck process starting from zero as

$$
X_{t}^{H}=v \int_{0}^{t} F(t, u) d B_{u}, \quad 0 \leq t \leq T,
$$

where $B$ is a standard Brownian motion with $B_{0}=0$, and for $0<u<t$

$$
\begin{aligned}
F(t, u)= & \left(H-\frac{1}{2}\right) \kappa_{H} e^{-t} u^{1 / 2-H} \\
& \times \int_{u}^{t} s^{H-1 / 2}(s-u)^{H-3 / 2} e^{s} d s,
\end{aligned}
$$

with $1 / 2<H<1, \kappa_{H}=(2 H \Gamma((3 / 2)-H) / \Gamma(H+(1 / 2)) \Gamma(2-$ $2 H))^{1 / 2}$, and

$$
\begin{aligned}
F(t, u)= & \kappa_{H} u^{1 / 2-H} \\
& \times\left(-e^{-t} \int_{u}^{t}(s-u)^{H-1 / 2} s^{H-1 / 2} e^{s} d s\right. \\
& +t^{H-1 / 2}(t-u)^{H-1 / 2}+\frac{2}{1-2 H} e^{-t} \\
& \left.\times \int_{u}^{t}(s-u)^{H-1 / 2} s^{H-3 / 2} e^{s} d s\right),
\end{aligned}
$$

with $0<H<1 / 2$.

For any $r, s \in[t, t+h]$ such that $r<s$, we have

$$
\begin{aligned}
\operatorname{Var}\left(\Delta X_{s}^{H} \mid \Delta X_{u}^{H}, u \leq r\right) & \geq \operatorname{Var}\left(\Delta X_{s}^{H}-\Delta X_{r}^{H} \mid B_{u}, u \leq r\right) \\
& =\operatorname{Var}\left(X_{s}^{H}-X_{r}^{H} \mid B_{u}, u \leq r\right) \\
& =\operatorname{Var}\left(X_{s}^{H} \mid B_{u}, u \leq r\right),
\end{aligned}
$$

where the last equality follows from the fact that $X_{r}^{H}$ is measurable with respect to $\sigma\left(B_{u}, u \leq r\right)$. Moreover, we can write

$$
X_{s}^{H}=v \int_{0}^{s} F(s, u) d B_{u}=v \int_{0}^{r} F(s, u) d B_{u}+v \int_{r}^{s} F(s, u) d B_{u} .
$$

Hence, by using the measurability of $\int_{0}^{r} F(s, u) d B_{u}$ with respect to $\sigma\left(B_{u}, u \leq s\right)$, we have

$$
\begin{aligned}
\operatorname{Var}\left(X_{s}^{H} \mid B_{u}, u \leq r\right) & =\operatorname{Var}\left(v \int_{r}^{s} F(s, u) d B_{u} \mid B_{u}, u \leq r\right) \\
& =\operatorname{Var}\left(v \int_{r}^{s} F(s, u) d B_{u}\right) \\
& \geq C_{H}(s-r)^{2 H},
\end{aligned}
$$

where, to obtain the second equality, we have used the fact that $\int_{r}^{s} F(s, u) d B_{u}$ is independent of $\sigma\left(B_{u}, u \leq s\right.$ ) (by the independence of the increments of the Brownian motion). Combining (31), inequation (35), and inequation (37), we have

$$
\operatorname{det} R\left(s_{1}, s_{2}, \ldots, s_{n}\right) \geq C_{H} \prod_{j=1}^{n}\left(s_{j}-s_{j-1}\right)^{2 H}>0,
$$


where $s_{0}=0$. Hence, the change of variable $V=R^{1 / 2} U, U=$ $\left(u_{1}, \ldots, u_{n}\right)$ implies that

$$
\begin{gathered}
\int_{\mathbb{R}^{n}} E\left(\exp \left(i \sum_{j=1}^{n} u_{j}\left(X_{s_{j}}^{H}-X_{t}^{H}\right)\right)\right) \prod_{j=1}^{n} d u_{j} \\
=\int_{\mathbb{R}^{n}} E\left(\exp \left(i \sum_{j=1}^{n} u_{j} \Delta X_{s_{j}}^{H}\right)\right) \prod_{j=1}^{n} d u_{j} \\
=\frac{(2 \pi)^{n / 2}}{\left(\operatorname{det} R\left(s_{1}, s_{2}, \ldots, s_{n}\right)\right)^{1 / 2}} .
\end{gathered}
$$

Hence,

$$
\begin{aligned}
E[L & (t+h, x)-L(t, x)]^{n} \\
= & \frac{n !}{(2 \pi)^{n / 2}} \\
& \times \int_{t<s_{1}<\cdots<s_{n}<t+h} \frac{1}{\left(\operatorname{det} R\left(s_{1}, s_{2}, \ldots, s_{n}\right)\right)^{1 / 2}} d s_{1} \cdots d s_{n} \\
\leq & C_{H} \frac{n !}{(2 \pi)^{n / 2}} \int_{t<s_{1}<\cdots<s_{n}<t+h} \prod_{j=1}^{n} \frac{1}{\left(s_{j}-s_{j-1}\right)^{H}} d s_{1} \cdots d s_{n} \\
\leq & C_{H} \frac{n !}{(2 \pi)^{n / 2}} h^{n(1-H)} \frac{(\Gamma(1-H))^{n}}{\Gamma(1+n(1-H))} .
\end{aligned}
$$

Following from Stirling's formula, we have $n ! / \Gamma(1+n(1-$ $H)) \leq A^{n} n !^{H}, n \geq 2$, for a suitable finite number $A$. So

$$
E\left(\frac{L(t+h, x)-L(t, x)}{h^{1-H}}\right)^{n} \leq C^{n} n !^{H} .
$$

Following, we first prove that for any $K>0$, there exists a positive and finite constant $B>0$, depending on $t$, such that for sufficiently small $u$

$$
P\left(L(t+h, x)-L(t, x) \geq \frac{B h^{1-H}}{u^{H}}\right) \leq e^{-(K / u)} .
$$

First consider $u$ of the form $u=1 / n$. By Chebyshev's inequality and inequation (41), we have

$$
\begin{aligned}
P( & \left.L(t+h, x)-L(t, x) \geq B h^{1-H} n^{H}\right) \\
& \leq E\left(\frac{L(t+h, x)-L(t, x)}{B h^{1-H} n^{H}}\right)^{n} \\
& \leq \frac{C^{n}}{B^{n}}\left(\frac{1}{n}\right)^{n H} n !^{H} \text { (by Stirling's formula) } \\
& \leq \frac{C^{n}}{B^{n}}(2 \pi n)^{H / 2} e^{-H n} \\
& =\exp \left(n\left(\log \left(\frac{C}{B}\right)-H\right)+\frac{H}{2}(\log n+\log 2 \pi)\right) .
\end{aligned}
$$

Choose $B>C$ and $n_{0}$ large such that for any $n \geq n_{0}$, to dominate (43) by $e^{-2 K n}$. Moreover, for $u$ sufficiently small, there exists $n \geq n_{0}$ such that $u_{n+1}<u<u_{n}$ and since $n \geq 1$, $n /(m+1) \geq 1 / 2$. This proves inequation (42).

On the other hand, if we take $u(h)=1 / \log \log (1 / h)$ and consider $h_{n}$ of the form $2^{-n}$, then inequation (42) implies

$$
\begin{aligned}
& P\left(L\left(t+h_{n}, x\right)-L(t, x) \geq B h_{n}^{1-H}\left(\log \log \left(\frac{1}{h_{n}}\right)\right)^{H}\right) \\
& \quad \leq n^{-2}
\end{aligned}
$$

for large $n$. So, following that Borel-Cantelli lemma and monotonicity arguments, we have

$$
\frac{L(t+h, x)-L(t, x)}{h^{1-H}} \leq B\left(\log \log \left(\frac{1}{h_{n}}\right)\right)^{H} \quad \text { a.s. }
$$

This completes the proof of inequation (28). we can obtain inequation (29) in the similar manner.

\section{Existence and Smoothness of Collision Local Time}

In this section we will study the so-called collision local time of two independent fractional Ornstein-Uhlenbeck $X^{H_{i}}=$ $\left\{X_{t}^{H_{i}}, t \geq 0\right\}, i=1,2$. It is defined formally by the following expression:

$$
\ell_{T}=\int_{0}^{T} \delta_{0}\left(X_{t}^{H_{1}}-X_{t}^{H_{2}}\right) d t
$$

where $\delta_{0}$ is the Dirac delta function. It is a measure of the amount of time for which the trajectories of the two processes, $X_{t}^{H_{1}}$ and $X_{t}^{H_{2}}$, collide on the time interval $[0, T]$. The collision local time for fractional Brownian motion has been studied by Jiang and Wang [27]. We shall show that the random variable $\ell_{T}$ exists in $L^{2}$. We approximate the Dirac delta function by the heat kernel

$$
p_{\varepsilon}(x)=\frac{1}{\sqrt{2 \pi \varepsilon}} e^{-x^{2} / 2 \varepsilon} \equiv \frac{1}{2 \pi} \int_{\mathbb{R}} e^{i x \xi} e^{-\varepsilon\left(\xi^{2} / 2\right)} d \xi .
$$

For $\varepsilon>0$ we define

$$
\begin{aligned}
\ell_{\varepsilon, T} & =\int_{0}^{T} p_{\epsilon}\left(X_{t}^{H_{1}}-X_{t}^{H_{2}}\right) d t \\
& =\frac{1}{2 \pi} \int_{0}^{T} \int_{\mathbb{R}} e^{i \xi\left(X_{t}^{H_{1}}-X_{t}^{H_{2}}\right)} \cdot e^{-\varepsilon\left(\xi^{2} / 2\right)} d \xi d t,
\end{aligned}
$$

and a natural question to study is that of the behavior of $\ell_{\varepsilon, T}$ as $\varepsilon$ tends to zero.

Theorem 3. For $H_{i} \in(0,1), i=1,2$. Then $\ell_{\varepsilon, T}$ converges in $L^{2}(\Omega, \mathscr{F}, P)$, as $\varepsilon \downarrow 0$. Moreover, the limit is denoted by $\ell_{T}$, then $\ell_{T} \in L^{2}(\Omega, \mathscr{F}, P)$. 
Proof. First we claim that $\ell_{\varepsilon, T} \in L^{2}(\Omega, \mathscr{F}, P)$ for every $\varepsilon>0$. By (48) we have

$$
\begin{gathered}
E\left(\ell_{\varepsilon, T}^{2}\right) \\
=\frac{1}{4 \pi^{2}} \iint_{0}^{T} \int_{\mathbb{R}^{2}} E e^{i \xi\left(X_{t}^{H_{1}}-X_{t}^{H_{2}}\right)+i \eta\left(X_{s}^{H_{1}}-X_{s}^{H_{2}}\right)} \\
\times e^{-\left(\left(\varepsilon\left(\xi^{2}+\eta^{2}\right)\right) / 2\right)} d \xi d \eta d s d t \\
=\frac{1}{4 \pi^{2}} \iint_{0}^{T} \int_{\mathbb{R}^{2}} e^{-(1 / 2) \sigma^{2}} e^{-\left(\left(\varepsilon\left(\xi^{2}+\eta^{2}\right)\right) / 2\right)} d \xi d \eta d s d t,
\end{gathered}
$$

where $\sigma^{2}$ denotes the variance of random variable $\xi\left(X_{t}^{H_{1}}-\right.$ $\left.X_{t}^{H_{2}}\right)+\eta\left(X_{s}^{H_{1}}-X_{s}^{H_{2}}\right)$, that is,

$$
\sigma^{2}:=\operatorname{Var}\left(\xi\left(X_{t}^{H_{1}}-X_{t}^{H_{2}}\right)+\eta\left(X_{s}^{H_{1}}-X_{s}^{H_{2}}\right)\right)
$$

According to the property of local nondeterminism (see Theorem 3.1 in [11]), we have

$$
\begin{aligned}
& \sigma^{2}= \operatorname{Var}\left(\xi\left(X_{t}^{H_{1}}-X_{s}^{H_{1}}\right)-\xi\left(X_{t}^{H_{2}}-X_{s}^{H_{2}}\right)\right. \\
&\left.+(\xi+\eta)\left(X_{s}^{H_{1}}-X_{s}^{H_{2}}\right)\right) \\
& \geq C[ \xi^{2}\left((t-s)^{2 H_{1}}+(t-s)^{2 H_{2}}\right) \\
&\left.+(\xi+\eta)^{2}\left(s^{2 H_{1}}+s^{2 H_{2}}\right)\right] .
\end{aligned}
$$

Thus, we have

$$
\begin{aligned}
& \frac{1}{4 \pi^{2}} \iint_{0}^{T} \int_{\mathbb{R}^{2}} e^{-(1 / 2) \sigma^{2}} e^{-\left(\varepsilon\left(\xi^{2}+\eta^{2}\right)\right) / 2} d \xi d \eta d s d t \\
& \leq \int_{0}^{T} \int_{0}^{t} \int_{\mathbb{R}^{2}} e^{-(C / 2)\left[\xi^{2}\left((t-s)^{2 H_{1}}+(t-s)^{2 H_{2}}\right)+(\xi+\eta)^{2}\left(s^{2 H_{1}}+s^{2 H_{2}}\right)\right]} d \xi d \eta d s d t \\
& =C \int_{0}^{T} \int_{0}^{t}\left[\left((t-s)^{2 H_{1}}+(t-s)^{2 H_{2}}\right)\right. \\
& \left.\quad \times\left(s^{2 H_{1}}+s^{2 H_{2}}\right)\right]^{-1 / 2} d s d t \\
& \leq C \int_{0}^{T} \int_{0}^{t}(t-s)^{-(1 / 2)\left(H_{1}+\mathrm{H}_{2}\right)} s^{-(1 / 2)\left(H_{1}+\mathrm{H}_{2}\right)} d s d t<\infty,
\end{aligned}
$$

because of $H_{i} \in(0,1)$, which yields

$$
E\left(\ell_{\varepsilon, T}^{2}\right)<\infty
$$

for all $\varepsilon \in(0,1]$.
Second, we claim that the sequence $\left\{\ell_{\varepsilon, T}, \varepsilon>0\right\}$ is of Cauchy in $L^{2}(\Omega, \mathscr{F}, P)$. For any $\theta, \varepsilon>0$ we have

$$
\begin{gathered}
E\left(\left|\ell_{\varepsilon, T}-\ell_{\theta, T}\right|^{2}\right) \\
=\frac{1}{4 \pi^{2}} \iint_{0}^{T} \int_{\mathbb{R}^{2}} E e^{i \xi\left(X_{t}^{H_{1}}-X_{t}^{H_{2}}\right)+i \eta\left(X_{s}^{H_{1}}-X_{s}^{H_{2}}\right)} \\
\cdot\left(e^{-(\varepsilon / 2) \xi^{2}}-e^{-(\theta / 2) \xi^{2}}\right) \\
\times\left(e^{-(\varepsilon / 2) \eta^{2}}-e^{-(\theta / 2) \eta^{2}}\right) d \xi d \eta d s d t \\
\leq \frac{1}{4 \pi^{2}} \sup _{\xi \in \mathbb{R}}\left(1-e^{-\left(\left(|\varepsilon-\theta|^{2}|\xi|^{2}\right) / 2\right)}\right)^{2} \\
\quad \times \iint_{0}^{T} \int_{\mathbb{R}^{2}} e^{-(1 / 2) \sigma^{2}} d \xi d \eta d s d t .
\end{gathered}
$$

Thus, dominated convergence theorem yields

$$
E\left(\left|\ell_{\varepsilon, T}-\ell_{\theta, T}\right|^{2}\right) \longrightarrow 0,
$$

as $\varepsilon \rightarrow 0$ and $\theta \rightarrow 0$, which leads to $\ell_{\varepsilon, T}$ is a Cauchy sequence in $L^{2}(\Omega, \mathscr{F}, P)$. Consequently, $\lim _{\varepsilon \rightarrow 0} \ell_{\varepsilon, T}$ exists in $L^{2}(\Omega, \mathscr{F}, P)$. This completes the proof.

For the increments of collision local time we have the following.

Theorem 4. Let $H_{1}, H_{2} \in(0,1)$ and $\beta=\min \left\{H_{1}, H_{2}\right\}$. Then the collision local time $\ell_{T}$ satisfies the following estimate:

$$
E\left(\left|\ell_{t}-\ell_{s}\right|^{2}\right) \leq C_{H_{1}, H_{2}}(t-s)^{2-2 \beta},
$$

for all $s, t, s<t$.

Proof. For any $0 \leq r, l \leq T$ we denote

$$
\sigma_{r, l}^{2}:=\operatorname{Var}\left(\xi\left(X_{r}^{H_{1}}-X_{r}^{H_{2}}\right)+\eta\left(X_{l}^{H_{1}}-X_{l}^{H_{2}}\right)\right) .
$$

Then the property of local nondeterminism (see Theorem 3.1 in [11]) yields

$$
\begin{array}{r}
\sigma_{r, l}^{2} \geq C\left[\xi^{2}\left((r-l)^{2 H_{1}}+(r-l)^{2 H_{2}}\right)\right. \\
\left.+(\xi+\eta)^{2}\left(l^{2 H_{1}}+l^{2 H_{2}}\right)\right]
\end{array}
$$

for a constant $C>0$. It follows from (48) that for $0 \leq s \leq t \leq$ $T$

$$
\begin{aligned}
& E\left(\left|\ell_{\varepsilon, t}-\ell_{\varepsilon, s}\right|^{2}\right) \\
& \quad=\frac{2}{(2 \pi)^{2}} \int_{s}^{t} \int_{s}^{r} d r d l \int_{\mathbb{R}^{2}} e^{-(1 / 2) \sigma_{r, l}^{2}} e^{-(\varepsilon / 2)\left(\xi^{2}+\eta^{2}\right)} d \xi d \eta \\
& \quad \leq C \int_{s}^{t} d r \int_{s}^{r}(r-l)^{-\beta} l^{-\beta} d l \\
& \quad \leq C(t-s)^{2-2 \beta} .
\end{aligned}
$$


Thus, Theorem 3 and Fatou's lemma yield

$$
\begin{aligned}
& E\left(\left|\ell_{t}-\ell_{s}\right|^{2}\right) \\
& \quad=E\left(\lim _{\varepsilon \rightarrow 0}\left|\ell_{\varepsilon, t}-\ell_{\varepsilon, s}\right|^{2}\right) \\
& \quad \leq \liminf _{\varepsilon \rightarrow 0} E\left(\left|\ell_{\varepsilon, t}-\ell_{\varepsilon, s}\right|^{2}\right) \leq C(t-s)^{2-2 \beta} .
\end{aligned}
$$

This completes the proof.

$$
\begin{aligned}
& \text { Let } \lambda_{t}=\operatorname{Var}\left(X_{t}^{H_{1}}-X_{t}^{H_{2}}\right) \text { for } t \geq 0 \text { and } \\
& \qquad \rho_{s, t}=E\left[\left(X_{t}^{H_{1}}-X_{t}^{H_{2}}\right)\left(X_{s}^{H_{1}}-X_{s}^{H_{2}}\right)\right],
\end{aligned}
$$

for $s, t \geq 0$.

Lemma 5 (An and Yan [28]). For any $x \in[-1,1)$ we have

$$
\sum_{n=1}^{\infty} \frac{(2 n-1) ! !}{(2 n-2) ! !} x^{n}=x(1-x)^{-3 / 2}
$$

where $(2 n-2) ! !=1 \cdot 3 \cdot 5 \cdots(2 n-1)$ and $(2 n-1) ! !=2 \cdot 4 \cdot$ $6 \cdots(2 n-2)$. Hence,

By Cauchy-Schwartz's inequality, we have $\rho_{s, t}^{2} \leq \lambda_{s} \lambda_{t}$.

$$
\begin{aligned}
\frac{\rho_{s, t}^{2}}{\left(\lambda_{s} \lambda_{t}-\rho_{s, t}^{2}\right)^{3 / 2}} & =\frac{\rho_{s, t}^{2}}{\lambda_{s} \lambda_{t}}\left(1-\frac{\rho_{s, t}^{2}}{\lambda_{s} \lambda_{t}}\right)^{-3 / 2}\left(\frac{1}{\lambda_{s} \lambda_{t}}\right)^{1 / 2} \\
& =\sum_{n=1}^{\infty} \frac{(2 n-1) ! !}{(2 n-2) ! !}\left(\frac{\rho_{s, t}^{2}}{\lambda_{s} \lambda_{t}}\right)^{n}\left(\frac{1}{\lambda_{s} \lambda_{t}}\right)^{1 / 2}
\end{aligned}
$$

for all $t, s \geq 0$ and $s \neq t$.

Below, we consider the smoothness of the collision local time. Our main object is to explain and prove the following theorem.

Theorem 6. Let $\ell_{T}, T \geq 0$ be the collision local time process of two independent fractional Ornstein-Uhlenbeck $X^{H_{i}}=$ $\left\{X_{t}^{H_{i}}, t \geq 0\right\}, i=1,2$, with respective indices $H_{i} \in(0,1)$. Then $\ell_{T}$ is smooth in the sense of the Meyer-Watanabe if and only if

$$
\min \left\{H_{1}, H_{2}\right\}<\frac{1}{3} \text {. }
$$

Proof. By Yan et al. [11], we have

$$
\begin{aligned}
\lambda_{t} \lambda_{s} & -\rho_{s, t}^{2} \\
\asymp & \left(s^{2 H_{1}}+s^{2 H_{2}}\right)\left(t^{2 H_{1}}+t^{2 H_{2}}\right) \\
& -\frac{1}{2}\left(t^{2 H_{1}}+s^{2 H_{1}}-|t-s|^{2 H_{1}}\right. \\
& \left.\quad+t^{2 H_{2}}+s^{2 H_{2}}-|t-s|^{2 H_{2}}\right) \\
\approx & \left(s^{2 H_{1}}+s^{2 H_{2}}\right)\left[(t-s)^{2 H_{1}}+(t-s)^{2 H_{2}}\right],
\end{aligned}
$$

where the notation $F \asymp G$ means that there are positive constants $c_{1}$ and $c_{2}$ so that

$$
c_{1} G(x) \leq F(x) \leq c_{2} G(x),
$$

in the common domain of definition for $F$ and $G$. Hence, following Theorem 2 in An and Yan [28], we have $\iint_{0}^{T}\left(\rho_{s, t}^{2} /\left(\lambda_{s} \lambda_{t}-\rho_{s, t}^{2}\right)^{3 / 2}\right) d s d t<\infty$ if and only if $\min \left\{H_{1}, H_{2}\right\}<1 / 3$. Therefore, in order to prove Theorem 6 , it only needs to prove: for $T \geq 0, \ell_{T}$ is smooth in the sense of the Meyer-Watanabe if and only if

$$
\iint_{0}^{T} \rho_{s, t}^{2}\left(\lambda_{t} \lambda_{s}-\rho_{s, t}^{2}\right)^{-3 / 2} d s d t<\infty
$$

In fact, for $\varepsilon>0, T \geq 0$ we denote

$$
\Theta_{\varepsilon}\left(u, T, \ell_{\varepsilon, T}\right):=E\left(\left|\Gamma_{\sqrt{u}} \ell_{\varepsilon, T}\right|^{2}\right),
$$

and $\Theta\left(u, T, \ell_{T}\right):=E\left(\left|\Gamma_{\sqrt{u}} \ell_{T}\right|^{2}\right)$. Thus, by Proposition 1 to prove that $(67)$ holds if and only if $\Phi_{\Theta}(1)<\infty$. Clearly, we have

$$
\begin{aligned}
\ell_{\varepsilon, T} & =\int_{0}^{T} p_{\varepsilon}\left(X_{t}^{H_{1}}-X_{t}^{H_{2}}\right) d t \\
= & \frac{1}{2 \pi} \int_{0}^{T} \int_{\mathbb{R}} e^{i \xi\left(X_{t}^{H_{1}}-X_{t}^{H_{2}}\right)} \cdot e^{-(1 / 2) \varepsilon^{2}} d \xi d t \\
= & \frac{1}{2 \pi} \int_{0}^{T} \int_{\mathbb{R}} e^{-(1 / 2) \xi^{2} \operatorname{Var}\left(X_{t}^{H_{1}}-X_{t}^{H_{2}}\right)} \\
\times e^{-(1 / 2) \varepsilon \xi^{2}} \sum_{n=0}^{\infty} i^{n} \sigma^{n}(t, \xi) H_{n} & \times\left(\frac{\xi\left(X_{t}^{H_{1}}-X_{t}^{H_{2}}\right)}{\sigma(t, \xi)}\right) d \xi d t \\
\equiv \sum_{n=0}^{\infty} F_{n} . &
\end{aligned}
$$

Notice that

$$
\begin{aligned}
& \Phi_{\Theta_{\varepsilon}}(1) \\
& =\sum_{n=0}^{\infty} n E\left(\left|F_{n}\right|^{2}\right) \\
& =\sum_{n=0}^{\infty} \frac{n}{4 \pi^{2}} \\
& \quad \times E\left[\iint_{0}^{T} \int_{\mathbb{R}^{2}} \exp \left(-\frac{1}{2} \varepsilon\left(|\xi|^{2}+|\eta|^{2}\right)\right)\right. \\
& \times \sigma^{n}(t, \xi) \sigma^{n}(s, \eta) \\
& \cdot \exp \left(-\frac{1}{2}\left(\xi^{2} \operatorname{Var}\left(X_{t}^{H_{1}}-X_{t}^{H_{2}}\right)\right.\right. \\
& \left.\left.\quad+\eta^{2} \operatorname{Var}\left(X_{s}^{H_{1}}-X_{s}^{H_{2}}\right)\right)\right)
\end{aligned}
$$




$$
\begin{aligned}
& H_{n}\left(\frac{\xi\left(X_{t}^{H_{1}}-X_{t}^{H_{2}}\right)}{\sigma(t, \xi)}\right) \\
&\left.\times H_{n}\left(\frac{\eta\left(X_{s}^{H_{1}}-X_{s}^{H_{2}}\right)}{\sigma(s, \eta)}\right) d \xi d \eta d s d t\right] \\
&=\sum_{n=1}^{\infty} \frac{1}{4 \pi^{2}(2 n-1) !} \times\left[\int_{0}^{T} \int_{\mathbb{R}^{2}}(\xi \eta)^{2 n}\right. \\
& \times\left[E\left(\left(X_{t}^{H_{1}}-X_{t}^{H_{2}}\right)\left(X_{s}^{H_{1}}-X_{s}^{H_{2}}\right)\right)\right]^{2 n} \\
& \quad \cdot \exp \left(-\left(\left(\xi^{2} \operatorname{Var}\left(\left(X_{t}^{H_{1}}-X_{t}^{H_{2}}\right)+\varepsilon\right)\right.\right.\right. \\
&+\eta^{2} \operatorname{Var}\left(\left(X_{s}^{H_{1}}-X_{s}^{H_{2}}\right)\right. \\
&\left.\left.\left.+\varepsilon)) \times \frac{1}{2}\right)\right) d \xi d \eta d s d t\right]
\end{aligned}
$$

for all $T \geq 0$, where we have used the following fact: For two random variables $X, Y$ with joint Gaussian distribution such that $E(X)=E(Y)=0$ and $E\left(X^{2}\right)=E\left(Y^{2}\right)=1$ we have (see, for example, Nualart [3])

$$
E\left(H_{n}(X) H_{m}(Y)\right)= \begin{cases}0, & m \neq n, \\ \frac{1}{n !}[E(X Y)]^{n}, & m=n\end{cases}
$$

We obtain

$$
\begin{aligned}
\Phi_{\Theta_{\varepsilon}}(1)= & \sum_{n=1}^{\infty} \frac{(\Gamma(n+1 / 2))^{2} 2^{2 n+1}}{4 \pi^{2}(2 n-1) !} \\
& \times \iint_{0}^{T} \frac{\rho_{s, t}^{2 n}}{\left(\left(\lambda_{s}+\varepsilon\right)\left(\lambda_{t}+\varepsilon\right)\right)^{n+(1 / 2)}} d s d t \\
= & \sum_{n=1}^{\infty} \frac{1}{2 \pi} \frac{(2 n-1) ! !}{(2 n-2) ! !} \\
& \times \iint_{0}^{T} \frac{\rho_{s, t}^{2 n}}{\left(\left(\lambda_{s}+\varepsilon\right)\left(\lambda_{t}+\varepsilon\right)\right)^{n+(1 / 2)}} d s d t \\
= & \frac{1}{2 \pi} \iint_{0}^{T} \frac{\rho_{s, t}^{2}}{\left(\left(\lambda_{s}+\varepsilon\right)\left(\lambda_{t}+\varepsilon\right)-\rho_{s, t}^{2}\right)^{3 / 2}} d s d t
\end{aligned}
$$

where we have used the following equality:

$$
\begin{aligned}
& \int_{\mathbb{R}} \xi^{2 n} \exp \left(-\frac{\xi^{2}\left(\operatorname{Var}\left(X_{t}^{H_{1}}-X_{t}^{H_{2}}\right)+\varepsilon\right)}{2}\right) d \xi \\
& \quad=2^{n+(1 / 2)} \Gamma\left(n+\frac{1}{2}\right)\left(\operatorname{Var}\left(X_{t}^{H_{1}}-X_{t}^{H_{2}}\right)+\varepsilon\right)^{-(n+(1 / 2))} .
\end{aligned}
$$

Hence, we have

$$
\lim _{\varepsilon \rightarrow 0} \Phi_{\Theta_{\varepsilon}}(1)=\frac{1}{2 \pi} \iint_{0}^{T} \frac{\rho_{s, t}^{2}}{\left(\lambda_{s} \lambda_{t}-\rho_{s, t}^{2}\right)^{3 / 2}} d s d t
$$

for all $T \geq 0$. This completes the proof.

\section{Acknowledgments}

The authors would like to thank anonymous earnest referee whose remarks and suggestions greatly improved the presentation of our paper. Guangjun Shen is partially supported by the National Natural Science Foundation of China (11271020), the Natural Science Foundation of Anhui Province (1208085MA11, 1308085QA14), and the Key Natural Science Foundation of Anhui Educational Committee (KJ2011A139), Dongjin Zhu is partially supported by the Key Natural Science Foundation of the Anhui Educational Committee (KJ2012ZD01, KJ2013A133) and the Philosophy and Social Science Planning Foundation of Anhui Province (AHSK11-12D128), and Yong Ren is partially supported by the National Natural Science Foundation of China (11371029).

\section{References}

[1] D. Revuz and M. Yor, Continuous Martingales and Brownian Motion, Springer, New York, NY, USA, 1999.

[2] J.-F. Gouyet, Physics and Fractal Structures, Springer, Berlin, Germany, 1996.

[3] D. Nualart, The Malliavin Calculus and Related Topics, Springer, New York, NY, USA, 2006.

[4] F. Biagini, Y. Hu, B. Øksendal, and T. Zhang, Stochastic Calculus for Fractional Brownian Motion and Applications, Springer, London, UK, 2008.

[5] Y. S. Mishura, Stochastic Calculus for Fractional Brownian Motion and Related Processes, vol. 1929 of Lecture Notes in Mathematics, Springer, Berlin, Germany, 2008.

[6] W. Willinger, M. S. Taqqu, and V. Teverovsky, "Stock market prices and long-range dependence," Finance Stochastics, vol. 3, no. 1, pp. 1-13, 1999.

[7] B. B. Mandelbrot and J. W. van Ness, "Fractional Brownian motions, fractional noises and applications," SIAM Review, vol. 10, pp. 422-437, 1968.

[8] P. Cheridito, H. Kawaguchi, and M. Maejima, "Fractional Ornstein-Uhlenbeck processes," Electronic Journal of Probability, vol. 8, article 3, pp. 1-14, 2003.

[9] S. C. Lim and S. V. Muniandy, "Generalized Ornstein-Uhlenbeck processes and associated self-similar processes," Journal of Physics A, vol. 36, no. 14, pp. 3961-3982, 2003.

[10] R. Metzler and J. Klafter, "The restaurant at the end of the random walk: recent developments in the description of anomalous transport by fractional dynamics," Journal of Physics A, vol. 37, no. 31, pp. R161-R208, 2004.

[11] L. Yan, Y. Lu, and Z. Xu, "Some properties of the fractional Ornstein-Uhlenbeck process," Journal of Physics A, vol. 41, no. 14, Article ID 145007, 17 pages, 2008.

[12] L. Yan and M. Tian, "On the local times of fractional OrnsteinUhlenbeck process," Letters in Mathematical Physics, vol. 73, no. 3, pp. 209-220, 2005. 
[13] S. R. S. Varadhan, "Appendix to euclidean quantum field theory by K. Symanzk," in Local Quantum Theory, R. Jost, Ed., Academic Press, New York, NY, USA, 1969.

[14] R. J. Adler, The Geometry of Random Fields, John Wiley \& Sons, New York, NY, USA, 1981.

[15] D. Geman and J. Horowitz, "Occupation densities," The Annals of Probability, vol. 8, no. 1, pp. 1-67, 1980.

[16] Y.Xiao, "Local times and related properties of multidimensional iterated Brownian motion," Journal of Theoretical Probability, vol. 11, no. 2, pp. 383-408, 1998.

[17] Y. Xiao, "Properties of local-nondeterminism of Gaussian and stable random fields and their applications," Annales de la Faculté des Sciences de Toulouse Série 6, vol. 15, no. 1, pp. 157193, 2006.

[18] Y. Xiao, "Hölder conditions for the local times and the Hausdorff measure of the level sets of Gaussian random fields," Probability Theory and Related Fields, vol. 109, no. 1, pp. 129-157, 1997.

[19] Y. Hu, "Hausdorff and packing measures of the level sets of iterated Brownian motion," Journal of Theoretical Probability, vol. 12, no. 2, pp. 313-346, 1999.

[20] B. Boufoussi, M. Dozzi, and R. Guerbaz, "On the local time of multifractional Brownian motion,” Stochastics, vol. 78, no. 1, pp. 33-49, 2006.

[21] B. Boufoussi, M. Dozzi, and R. Guerbaz, "Sample path properties of the local time of multifractional Brownian motion," Bernoulli, vol. 13, no. 3, pp. 849-867, 2007.

[22] S. M. Berman, "Local nondeterminism and local times of Gaussian processes," Indiana University Mathematics Journal, vol. 23, pp. 69-94, 1974.

[23] J. P. Nolan, "Local nondeterminism and local times for stable processes," Probability Theory and Related Fields, vol. 82, no. 3, pp. 387-410, 1989.

[24] Y. Hu, "Self-intersection local time of fractional Brownian motions - via chaos expansion," Journal of Mathematics of Kyoto University, vol. 41, no. 2, pp. 233-250, 2001.

[25] S. Watanabe, Stochastic Differential Equation and Malliavin Calculus, Tata Institute of Fundamental Reaearch, Springer, New York, NY, USA, 1984.

[26] S. M. Berman, "Local times and sample function properties of stationary Gaussian processes," Transactions of the American Mathematical Society, vol. 137, pp. 277-299, 1969.

[27] Y. Jiang and Y. Wang, "On the collision local time of fractional Brownian motions," Chinese Annals of Mathematics B, vol. 28, no. 3, pp. 311-320, 2007.

[28] L. An and L. Yan, "Smoothness for the collision local time of fractional Brownian motion," Preprint. 


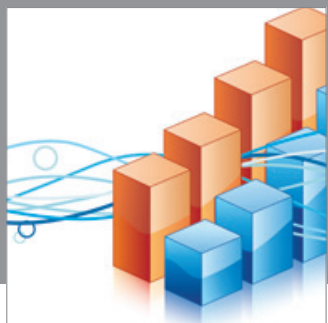

Advances in

Operations Research

mansans

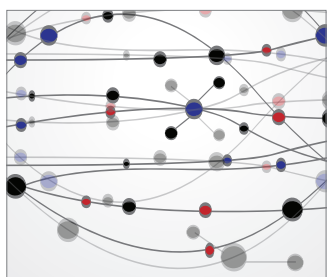

The Scientific World Journal
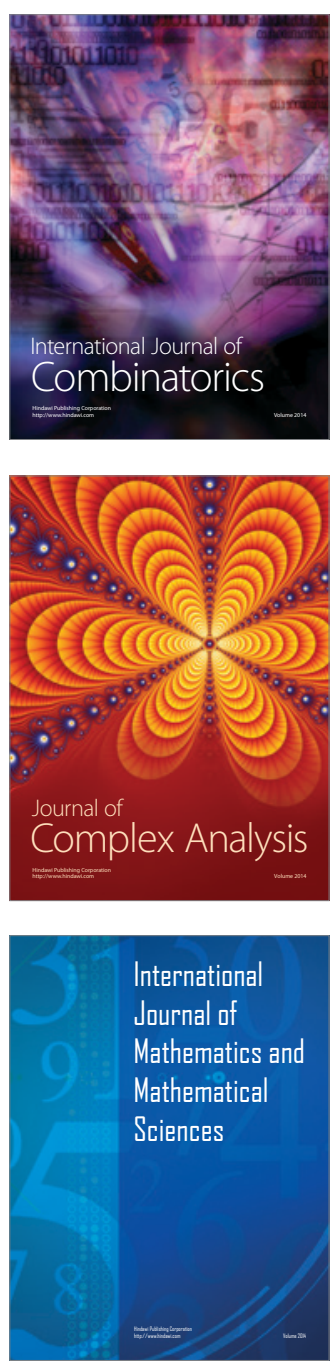
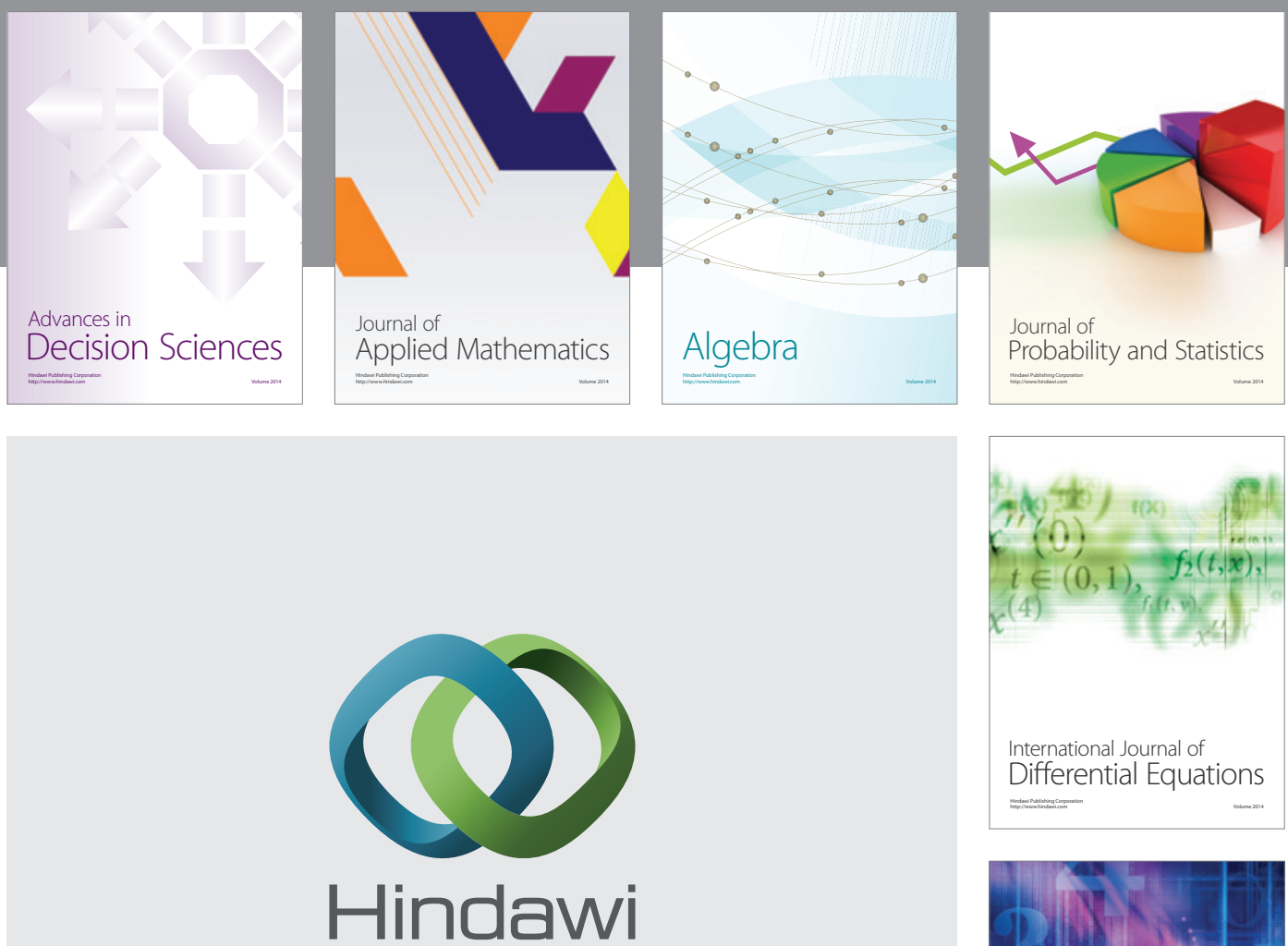

Submit your manuscripts at http://www.hindawi.com
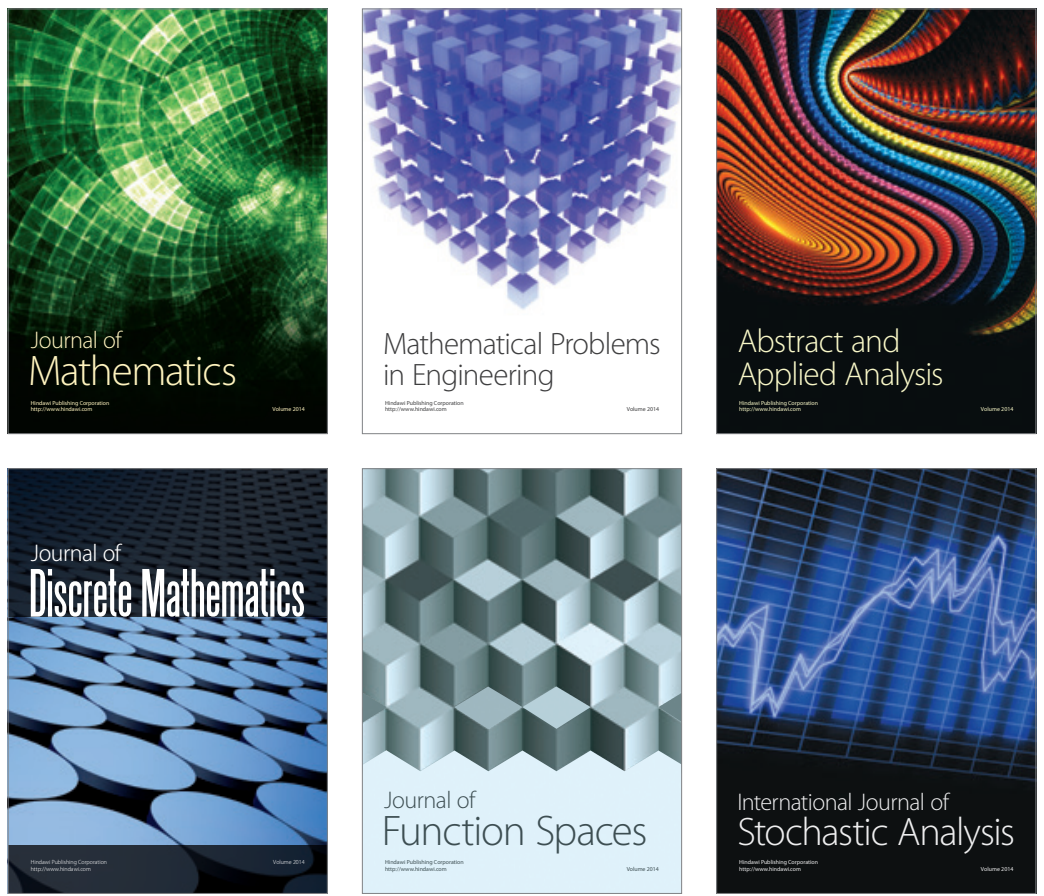

Journal of

Function Spaces

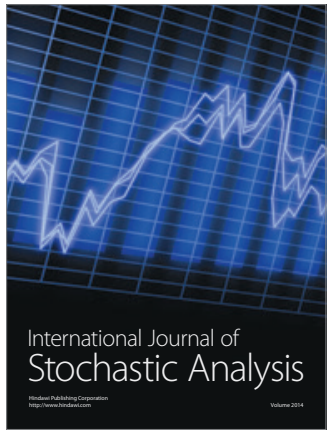

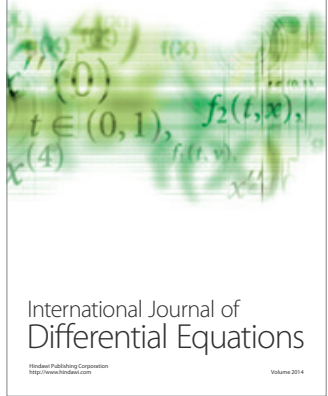
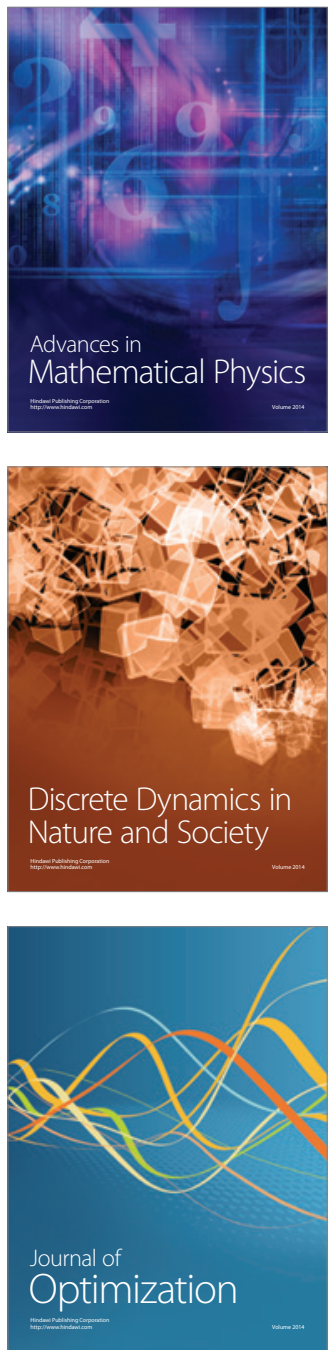